\title{
The CFTR Corrector, VX-809 (Lumacaftor), Rescues ABCA4 Trafficking Mutants: a Potential Treatment for Stargardt Disease
}

\author{
Qiangni Liu Inna Sabirzhanova Emily Anne Smith Bergbower Murali Yanda \\ William G. Guggino Liudmila Cebotaru \\ Division of Gastroenterology and Hepatology, Departments of Medicine, Johns Hopkins University, \\ Baltimore, MD, USA
}

\section{Key Words}

Stargardt disease $\cdot$ Common mutations $\bullet$ Rescue $\cdot$ Chaperones $\bullet$ Correctors

\begin{abstract}
Background/Aims: Mutations in ABCA4 cause Stargardt macular degeneration, which invariably ends in legal blindness. We studied two common mutants, A1038V (in NBD1) and G1961E (in NBD2), with the purpose of exploring how they interact with the cell's quality control mechanism. The study was designed to determine how these mutants can be rescued. Methods: We expressed wt and mutant ABCA4 in HEK293 cells and studied the effect of the mutations on trafficking and processing and the ability of correctors to rescue them. We used a combination of western blotting, confocal microscopy and surface biotinylation coupled with pulldown of plasma membrane proteins. Results: G1961E is sensitive to inhibitors of the aggresome, tubacin and the lysosome, bafilomycin A. Both mutants cause a reduction in heat shock protein, Hsp27. Incubation of HEK293 cells expressing the mutants with VX-809, an FDA approved drug for the treatment of cystic fibrosis, increased the levels of A1038V and G1961E by 2- to 3-fold. Importantly, VX-809 increased the levels of both mutants at the plasma membrane suggesting that trafficking had been restored. Transfecting additional Hsp27 to the cells also increased the steady state levels of both mutants. However, in combination with VX-809 the addition of Hsp27 caused a dramatic increase in the protein expression particularly in the G1961 mutant which increased approximately 5-fold. Conclusion: Our results provide a new mechanism for the rescue of ABCA4 trafficking mutants based on the restoration of Hsp27. Our results provide a pathway for the treatment of Stargardt disease.
\end{abstract}




\section{Cellular Physiology Cell Physiol Biochem 2019;53:400-412 \\ \begin{tabular}{ll|l} 
and Biochemistry & $\begin{array}{l}\text { DOI: 10.33594/000000146 } \\
\text { Published online: } 13 \text { August } 2019\end{array}$ & $\begin{array}{l}\text { C } 2019 \text { The Author(s). Published by } \\
\text { Cell Physiol Biochem Press GmbH\&Co. KG }\end{array}$ \\
\hline
\end{tabular} \\ Liu et al.: Rescuing ABCA4 Trafficking Mutants}

\section{Introduction}

ABCA4, commonly known as RIM protein [1], is a photoreceptor-associated protein that transports retinylidene-phosphatidyl ethanolamine, which facilitates the recycling of the all-trans retinal that is generated during the light cycle [2]. Mutations in $A B C A 4$ cause Stargardt disease, a common form of early-onset macular degeneration that results in poor central vision and impaired dark adaptation [3]. Failure to recycle all-trans retinal, resulting from mutations in $A B C A 4$, leads to the accumulation of lipofucsin in the retinal pigmented epithelium (RPE) and photoreceptor degeneration [4]. At present, there is no known cure for Stargardt disease; however, we have previously shown that, given the sequence similarity within the nucleotide binding domains of ABCA4 and ABCC7 (CFTR), small-molecule correctors designed to rescue processing mutants of CFTR can potentially also rescue carefully selected processing mutants of ABCA4 [5].

Orkambi, which contains the small-molecule corrector VX-809 in combination with a potentiator (VX-770), has been approved by the FDA to treat cystic fibrosis (CF), an autosomal recessive disease associated with defective fluid transport across several mucosal membranes throughout the body [6]. Orkambi is approved for the treatment of CF patients with the most common mutation, $\triangle$ F508-CFTR, which is well known to be a processing mutant [7]. In the case of Stargardt disease, over 500 mutations have been identified in $A B C A 4$, many of which lead to defective processing [8]. Given that CFTR and ABCA4 share common motifs [5] and that the corrector VX-809 has been shown to be safe and effective in many CF patients, we asked whether correctors that rescue $\triangle F 508$-CFTR could also rescue two common mutations in ABCA4, A1038V in the NBD1 domain and G1961E in the NBD2 domain. Importantly, they account for a large percentage of Stargardt patients (18.5\%) [9, 10]. We found that VX-809 could indeed increase the steady protein levels of both disease causing mutations. We also found that the heat shock protein, Hsp27, protein levels are reduced by these two mutants and that restoration of Hsp27 rescues the mutants. We found an additive effect by restoring Hsp27 protein levels in combination with VX-809. Our results provide a potential path forward for the development of a therapy for patients with Stargardt disease.

\section{Materials and Methods}

Generation of stably transfected HEK 293 cell lines

$A B C A 4$ cDNA expressed in the pCMV6-AC-GFP vector was purchased from OriGene Technologies. Two ABCA4 mutations, A1038V and G1961E, were generated using a QuikChange Lightning SiteDirected Mutagenesis Kit (Agilent Technologies) [5]. Flp-In human embryonic kidney (HEK) 293 cells (Catalog\#CRL-1573, Life Technologies) were cultured in Dulbecco's minimal essential medium (DMEM) (Life Technologies) containing 10\% fetal bovine serum (FBS), with penicillin $(100 \mathrm{U} / \mathrm{ml})$, streptomycin $(100 \mu \mathrm{g} / \mathrm{ml})$, and Zeocin $(100 \mu \mathrm{g} / \mathrm{mL})$ at $37^{\circ} \mathrm{C}$ in $5 \% \mathrm{CO}_{2}$. The stable cell lines were generated as described previously [5]. We would state that all experiments were conducted with GFP tagged ABCA4. In our past experiments tagged CFTR behaved similarly to untagged versions [11-13].

\section{Biotinylation}

As described previously [5], HEK 293 cells stably expressing ABCA4 mutants A1038V or G1961E were exposed to sulfo-NHS-SS-biotin (Thermo Scientific) for $30 \mathrm{~min}$ on ice, rinsed three times with glycine quenching buffer (200 mM glycine and $25 \mathrm{mM}$ Tris/ $\mathrm{HCl}$ [pH 8.0] in DPBS with calcium and magnesium), and solubilized in lysis buffer (150 mM NaCl, $50 \mathrm{mM}$ Tris/HCl, 1\% NP-40, and protease inhibitors).

\section{Western blotting}

Protein samples were electrophoresed on SDS/PAGE (7.5\% gels) and transferred to PVDF membranes. Membranes were blocked with 5\% non-fat dried skimmed milk in TBST Tris-buffered saline (TBST: $50 \mathrm{mM}$ Tris and $150 \mathrm{mM} \mathrm{NaCl}[\mathrm{pH} 8.0]$ with $0.05 \%$ Tween 20) for $1 \mathrm{~h}$, then incubated overnight at $4^{\circ} \mathrm{C}$ with an 


\section{Cellular Physiology Cell Physiol Biochem 2019;53:400-412 \\ \begin{tabular}{ll|l} 
and Biochemistry & $\begin{array}{l}\text { DOl: 10.33594/000000146 } \\
\text { Published online: } 13 \text { August } 2019\end{array}$ & $\begin{array}{l}\text { O } 2019 \text { The Author(s). Published by } \\
\text { Cell Physiol Biochem Press GmbH\&Co. KG }\end{array}$ \\
\cline { 2 - 3 }
\end{tabular} \\ Liu et al.: Rescuing ABCA4 Trafficking Mutants}

anti-ABCA4 antibody (sc-65672, Santa Cruz Biotechnology). Ezrin (sc-58758, Santa Cruz Biotechnology) detection was used as a loading control. The blots were then washed and incubated with sheep anti-mouse IgG peroxidase-linked antibody (GE Healthcare). Immunoreactive bands were visualized by SuperSignal West Dura substrate (Thermo Scientific) using a Fuji Film LAS-4000 Plus system with a cooled CCD camera. Image Gauge version 3.2 software (Fuji Film) was used for quantification of the bands. Background was determined in the vicinity of each of the band quantified and the background values subtracted. For experiments involving lysate samples, 80-100 $\mu \mathrm{g}$ of protein was loaded into the gel. For surface biotinylation, 3000-3500 $\mu \mathrm{g}$ was loaded.

\section{Small-molecule corrector and inhibitors}

The small-molecule corrector VX-809 was obtained from the Selleck Chemicals. The effect of proteasome, aggresome, and autophagosome inhibitors (MG132, tubacin, and bafilomycin $\mathrm{A}_{1}$, respectively) on the mutants was also studied at the concentrations specified (see [5] for details).

\section{Immunofluorescence and Fluorescent Microscopy}

A Zeiss LSM 510 laser scanning system and 63x oil-immersion lens were used. Cells were seeded onto cover glasses in 6 well plates for 24 hours. If cells were treated with VX-809, treatment was for 12 hours prior to fixation. The following steps were done at room temperature: cells were fixed with $4 \%$ formaldehyde for 15 minutes. Subsequently, cells were permeabilized with 0.3\% Triton X-100 in DPBS for 5-7 minutes and blocked with $3 \%$ bovine albumin serum (BSA) for 45 minutes. After blocking, cells were incubated with primary antibodies and then with secondary antibodies conjugated to Alexa 594 or Alexa 647. Cells were incubated with a 1:1000 dilution of DAPI for 5 minutes and mounted using ProLong Gold Antifade (Invitrogen). Images were cropped, adjusted, and exported as TIFs using Imaris Imaging Software. Figures for publishing were put together using Adobe Photoshop. For quantification of colocalization, Pearson's correlation coefficients were calculated using Imaris Imaging Software.

\section{Results}

\section{A1038V and G1961E are processing mutants of ABCA4}

Many genetic mutations that cause human disease result in misfolded proteins that are prematurely targeted for degradation by the ER-associated quality control pathways [14]. Here, we focused on two common Stargardt disease-causing mutations in $A B C A 4, \mathrm{~A} 1038 \mathrm{~V}$ and G1961E [15]. These mutations are in the NBD1 and 2 domains, respectively, and express much less protein (Fig. 1A \& B) than does wild-type (wt) ABCA4, indicating that these are likely processing mutants [5]. The mutants also significantly reduce the steady state levels of heat shock protein, Hsp27, (Fig. 1A \& C) indicating that they are engaging the cell's quality control mechanism.

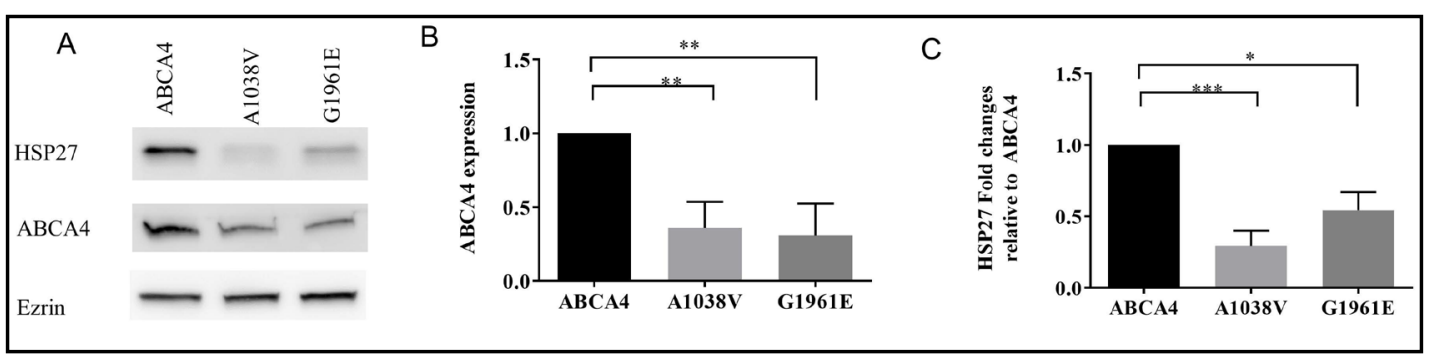

Fig. 1. Wild type (wt) and mutant ABCA4 express less protein and reduce Hsp27. (A \&B) HEK-293 cell lines stably expressing A1038V or G1961E show reduced expression of ABCA4 when compared to wt ABCA4. $n=5$. (C) Note the profound decrease in steady state levels of Hsp27 by the mutants. (A) Ezrin was used as the loading control. Protein expression of A1038V and G1961E is significantly lower than that of wt ABCA4. 
In order to determine whether the protein products of these two mutants are degraded in the cell, we treated HEK 293 cells expressing the mutants with MG132, a non-specific inhibitor of proteasomal degradation. Interestingly, the steady-state levels of A1038V were decreased (Fig. 2A), whereas those of G1961E (Fig. 2B) were increased following inhibition of proteasomal degradation. Next, we treated the cells with tubacin, an HDAC6 inhibitor that is involved in trafficking mutant proteins for degradation in the aggresome [16]. Cells treated with tubacin showed an increase in the steady-state protein levels of both mutants, but the magnitude of the effect differed greatly between the two. The effect on A1038V (Fig. 3A) was approximately 2 -fold, whereas the steady state levels of G1961E (Fig. 3B) were increased dramatically following tubacin by approximately 3.5 fold. Finally, we treated the cells with bafilomycin A1, a proton pump inhibitor that blocks lysosomal degradation [11]. This inhibitor caused increases in the steady-state levels of both proteins (Fig. 4A \& B), particularly the steady state levels of G1961E (Fig. 4B) which increased 2-fold. The data therefore show that these two mutants in NBD1 and 2, respectively, have very different responses to these inhibitors. The data also suggests that both mutants are misfolded

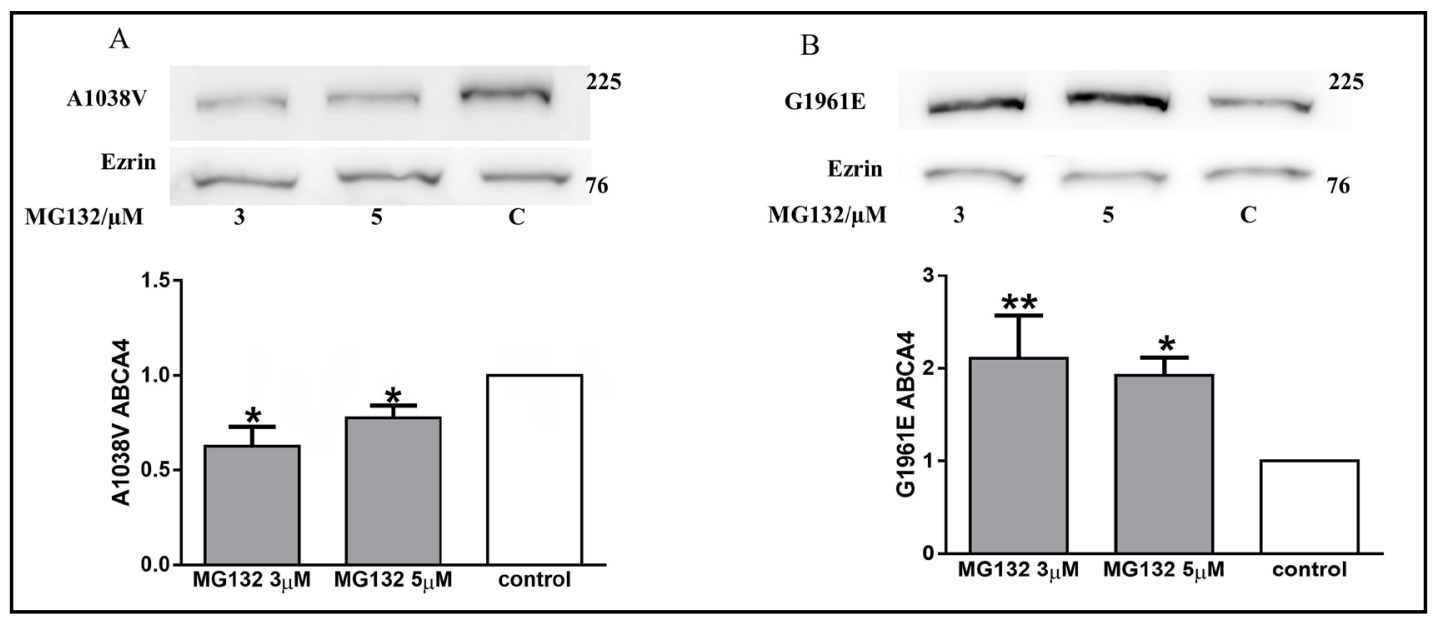

Fig. 2. Proteasomal degradation pathway. HEK-293 cell lines stably expressing (A) A1038V, or (B) G1961E were treated for $16 \mathrm{~h}$ with increasing doses of proteasome inhibitor MG-132. Data are normalized to the $0 \mu \mathrm{M}$ control. ${ }^{*} \mathrm{P}<0.05$. $\mathrm{n}=4$. Ezrin was used as the loading control. Mutations A1038V and G1961E are degraded to some extent in the proteasome.

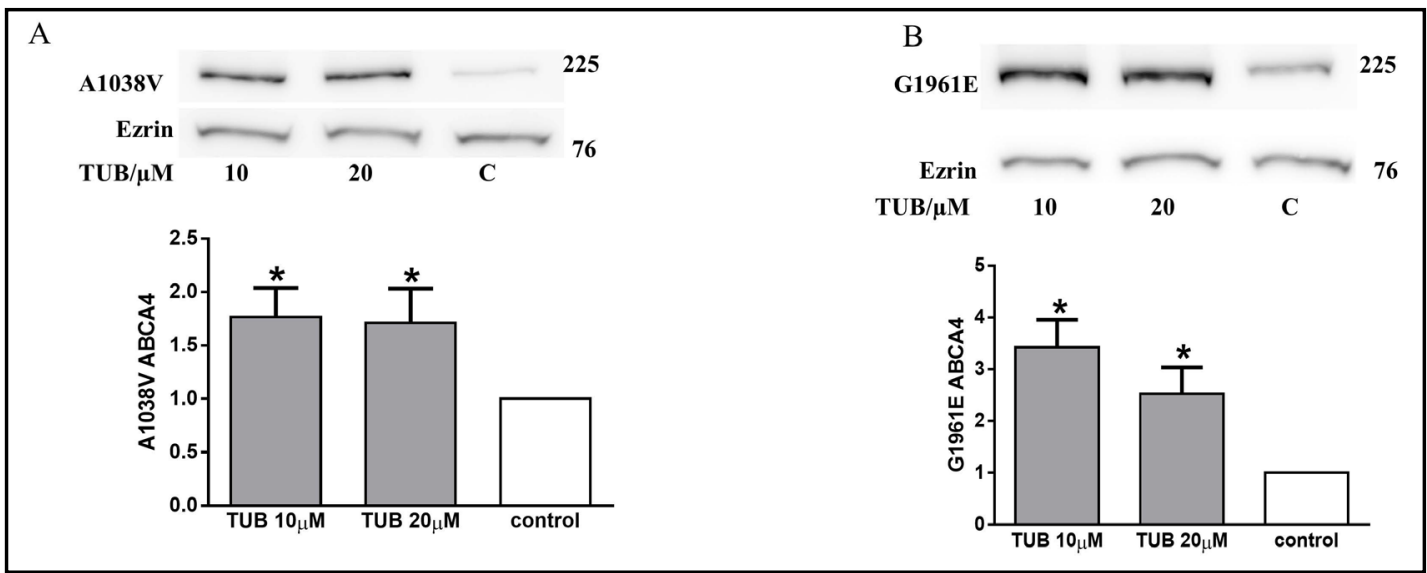

Fig. 3. Aggresomal degradation pathway. HEK-293 cell lines stably expressing (A) A1038V, or (B) G1961E were treated for $16 \mathrm{~h}$ with increasing doses of the aggresome inhibitor tubacin. Data are normalized to the $0 \mu \mathrm{M}$ control. ${ }^{*} \mathrm{P}<0.05 . \mathrm{n}=3-4$. Ezrin was used as the loading control. The A1038 V and G1961E mutants are significantly degraded in the aggresome. 


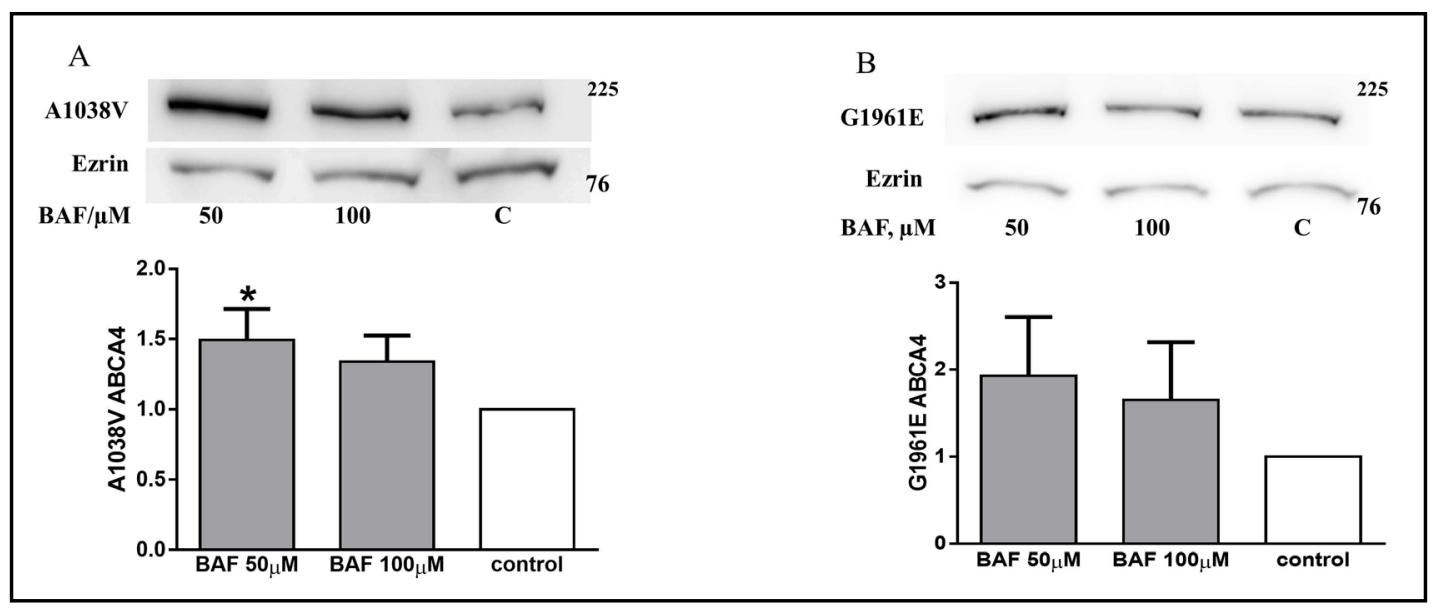

Fig. 4. Lysosomal degradation pathway. HEK-293 cell lines stably expressing (A) A1038V, or (B) G1961E were treated for $16 \mathrm{~h}$ with increasing doses of a proton pump inhibitor of lysosome degradation, bafilomycin A. Data are normalized to the $0 \mu \mathrm{M}$ control. ${ }^{*} \mathrm{P}<0.05$. $\mathrm{n}=3-4$. Ezrin was used as the loading control. The A1038V mutant is degraded in the lysosome, but G1961E is not.

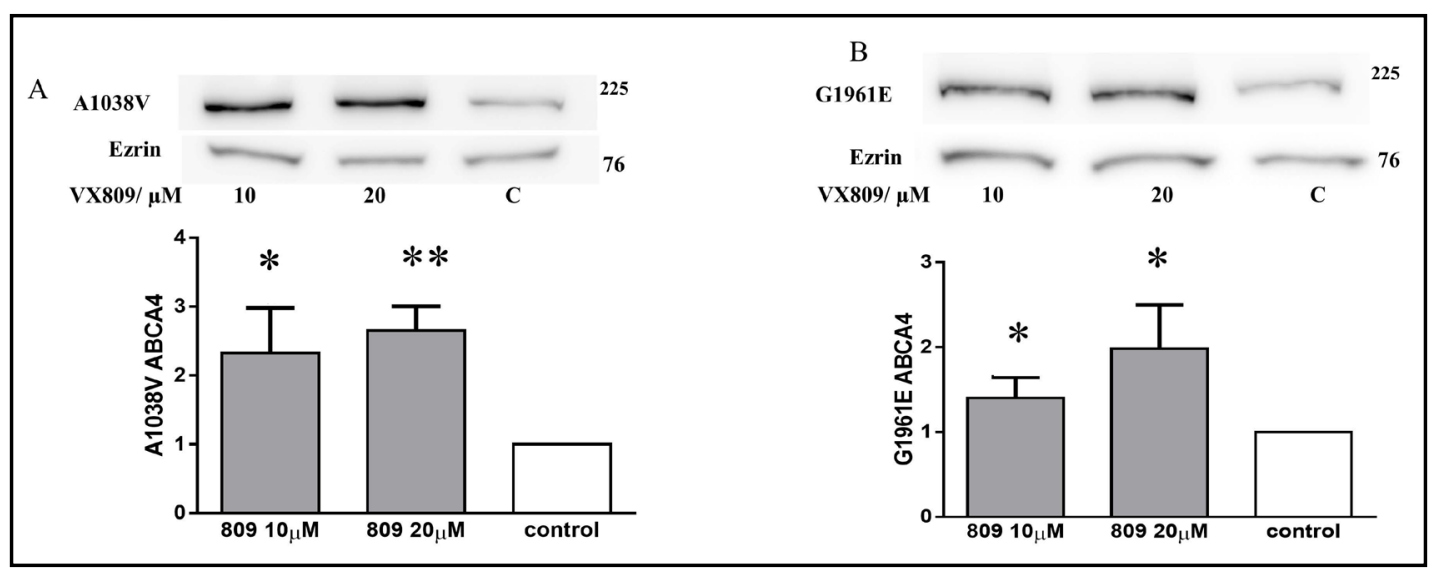

Fig. 5. Effect of VX-809 on the mutants A1038V and G1961E. The HEK-293 cell line stably expressing (A) A1038V, or (B) G1961E was treated with VX-809 for $16 \mathrm{~h}$. Data are normalized to the $0 \mu \mathrm{M}$ control. ${ }^{*} \mathrm{P}<0.05$. $\mathrm{n}=3-4$. The protein expression of ABCA4 mutants A1038V, G1961E is significantly increased after treatment with VX809.

and engage the cell's quality control mechanism in different ways. A1038V and G1961E are degraded by the aggresome, whereas G1961E is degraded both by the aggresome and proteasome.

\section{ABCA4 mutants are rescued by VX-809}

As mentioned above, VX-809, discovered by Vertex Pharmaceuticals [17], has been approved for patients in a combination formulation with the potentiator VX-770 that is referred to as Orkambi [6]. We applied VX-809 (Fig. 5A \& B) and noted a robust response that increased the steady-state levels of both mutants 2- to 3-fold. Importantly, VX-809 significantly increased the surface expression of both mutants (Fig. 6A), as measured by surface biotinylation, with the greatest effect being a doubling of the surface expression of G1961E (Fig. 6A). Cell lysates are depicted for comparison (Fig. 6B).

Given that ABCA4 functions at the plasma membrane it is important to determine the extent to which surface expression of the mutants is enhanced by VX-809. Although we showed in Fig. 6 that surface expression of VX-809 is increased, we extended this observation by evaluating the surface expression of ABCA4 using confocal microscopy. We 


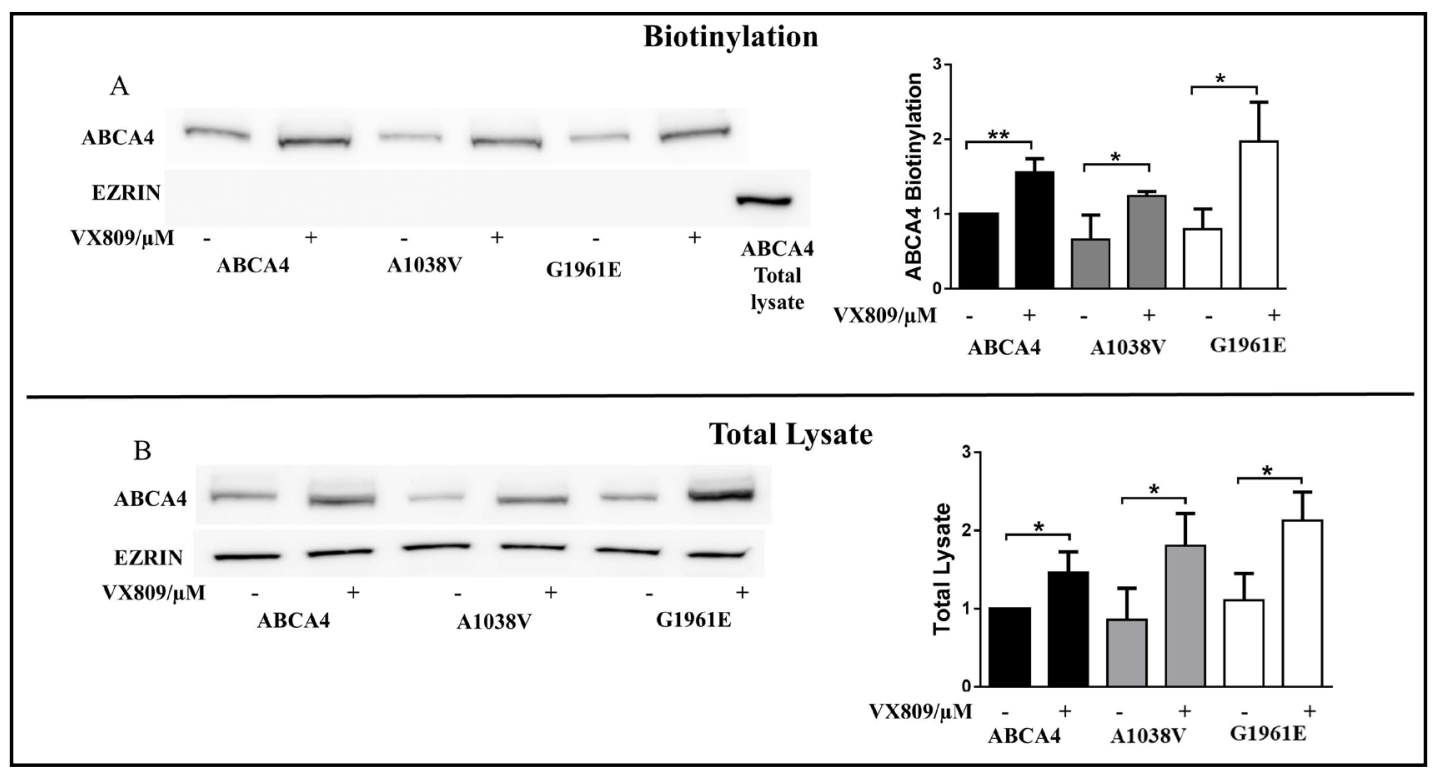

Fig. 6. Effect of VX-809 on the surface expression of the mutants. Treatment with VX-809 produced an increase in protein expression at the cell surface (A). Ezrin was used as an internal control. Note that ezrin is an intracellular protein, and the failure to detect any ezrin in the biotinylated fraction demonstrates convincingly that the plasma membrane was intact during the biotinylation experiment. The surface protein expression of ABCA4 and the mutants A1038V and G1961E are significantly increased after treatment with VX809. (B) total lysate values are shown for comparison.

marked the plasma membrane using a fluorescent approach, whereby we visualized the expression of cadherin a marker for the plasma membrane and looked for colocalization with ABCA4. Fig. 7 shows that wt-ABCA4 colocalizes nicely with cadherin demonstrating that it is indeed located at the plasma membrane. Its membrane localization is not affected by treatment with VX-809. Fig. 8 \& 9 shows that VX-809 restores the membrane localization of both mutants. The surface biotinylation and confocal microscopy experiments agree that rank order of the effect of VX-809 is G1961E >A1038V > ABCA4. Given that ABCA4 functions at the plasma membrane these data add more evidence that VX-809 maybe therapeutic for Stargardt disease.

\section{Restoring Hsp27 levels}

As noted in Fig. 1 there is a large drop in the steady state levels of Hsp27 when either A1038V or G1961E are expressed in the HEK293 cells compared to wt-ABCA4. In order to determine whether this reduction plays a role in the processing of ABCA4 mutants we transfected additional Hsp27 into the cells to raise the steady state level of expression. Fig. $10 \mathrm{~A}$ shows that increasing the expression of Hsp27, has a greater effect on increasing the steady state levels of wt-ABCA4 than treating cells with VX-809. Increasing Hsp27 expression in the presence of VX-809 is additive causing a dramatic two- fold increase in wt-ABCA4. Fig. 10B shows that VX-809 alone has no effect on Hsp27 levels. The levels are increased dramatically following transfection of additional Hsp27 (Fig. 10B).

The results for the protein product of the mutant A1038V show a similar pattern. Fig. 11A shows that increasing Hsp27, has a greater effect on increasing the steady state levels of A1038V ABCA4 than treating cells with VX-809. Increasing Hsp27 levels in the presence of VX-809 is additive causing a dramatic three- fold increase in A1038V. Fig. 11B shows the Hsp27 controls. 


\section{Cellular Physiology and Biochemistry}

Fig. 7. Plasma membrane localization of WT-ABCA4 protein. (A) WT ABCA4-GFP (green) stably transfected cells were plated on coverslips. Cells were subsequently fixed and stained with DAPI (blue, nucleus) and antibodies against cadherin (red, plasma membrane). Areas of colocalization give off a yellow signal (white arrows). Representative extended focus images are shown. (B): Cell surface expression of ABCA4 protein is unaffected by VX-809. WT ABCA4GFP (green) stably transfected cells were plated on coverslips. Cells were subsequently fixed and stained with DAPI (blue, nucleus) and antibodies against cadherin (purple, plasma membrane). Areas of colocalization give off a white signal (white arrows). 3 separate

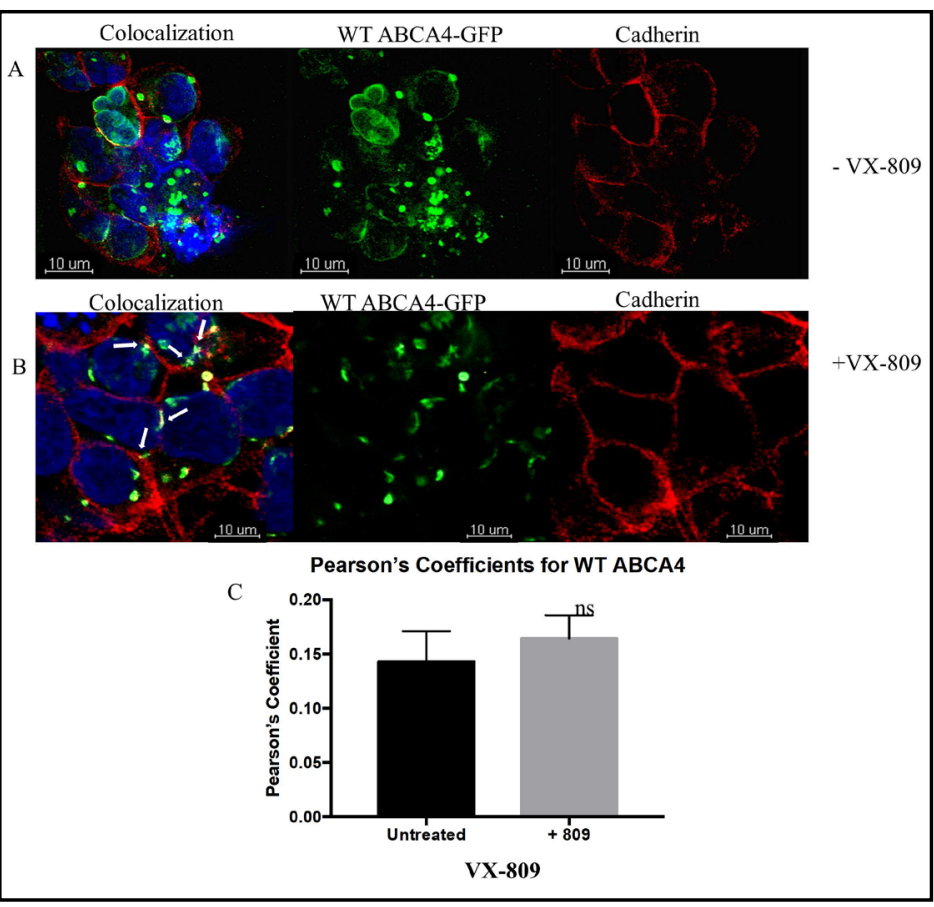
experiments were performed.

Representative extended focus images are shown. (C): Pearson's coefficients were obtained for VX-809 treated and untreated cells. Coefficients were compared using the student's t-test and found to be nonsignificant $(n=3)$.

Fig. 8. Mutant A1038V does not colocalize with cadherin at the plasma membrane but is rescued by VX-809. (A) A1038V ABCA4GFP (green) stably transfected cells were plated on coverslips. Cells were subsequently fixed and stained with DAPI (blue, nucleus) and antibodies against cadherin (red, plasma membrane). Representative extended focus images are shown. (B) Mutant A1038V shows cell surface expression when treated with VX-809. A1038V ABCA4-GFP (green) stably transfected cells were plated on coverslips. Cells were subsequently fixed and stained with DAPI (blue, nucleus)

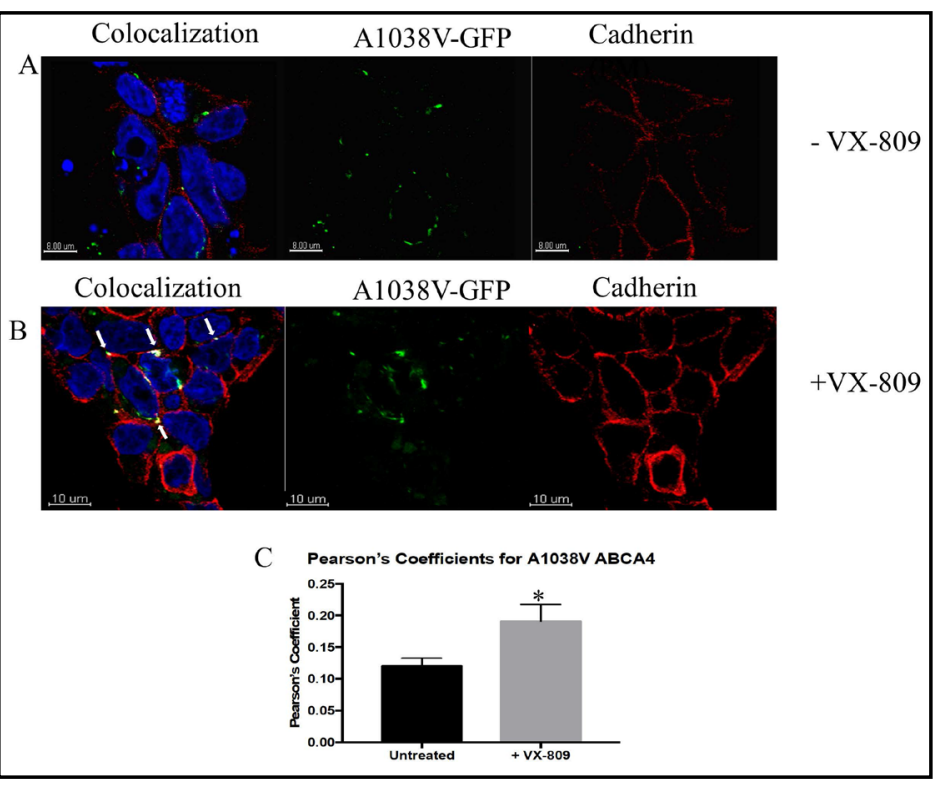
and antibodies against cadherin (purple, plasma membrane). Colocalization signal is white (white arrows). 3 separate experiments were performed. Representative extended focus images are shown. (C): Pearson's coefficients were obtained for 809 treated and untreated cells. Coefficients were compared using the student's t-test and found to be significant. Conclusion: VX-809 restores surface expression of the mutant A1038V. 
Fig. 9. Mutant G1961E does not colocalize at the plasma membrane but is rescued by VX-809 (A) G1961E ABCA4-GFP (green) stably transfected cells were plated on coverslips. Cells were subsequently fixed and stained with DAPI (blue, nucleus) and antibodies against cadherin (red, plasma membrane). Representative extended focus images are shown. (B): Mutant G1961E is rescued to the plasma membrane following treatment with VX-809. G1961E ABCA4GFP (green) stably transfected cells were plated on coverslips. Cells were subsequently fixed and stained with DAPI (blue, nucleus) and antibodies against cadherin (purple, plasma membrane). 3 separate experiments were performed. Representative

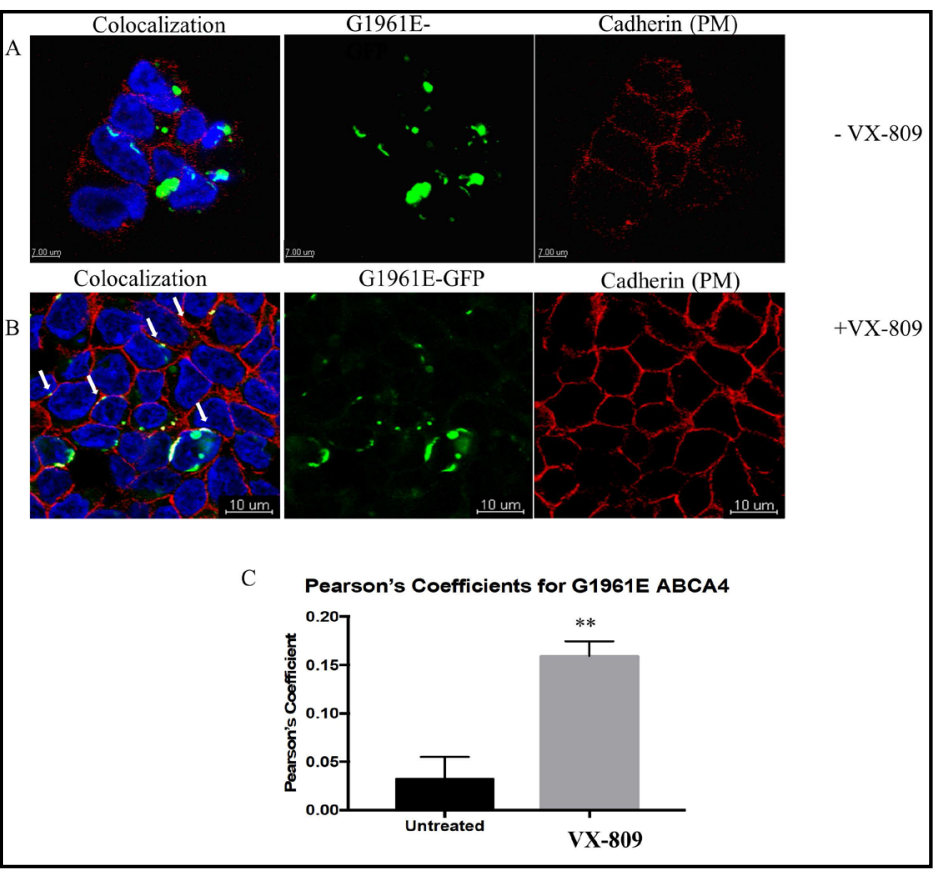
extended focus images are shown. Colocalization signal is white (white arrows). (C): Pearson's coefficients were obtained for VX-809 treated and untreated cells. Coefficients were compared using the student's t-test and found to be significant. Conclusion: VX-809 restores surface expression of the mutant.

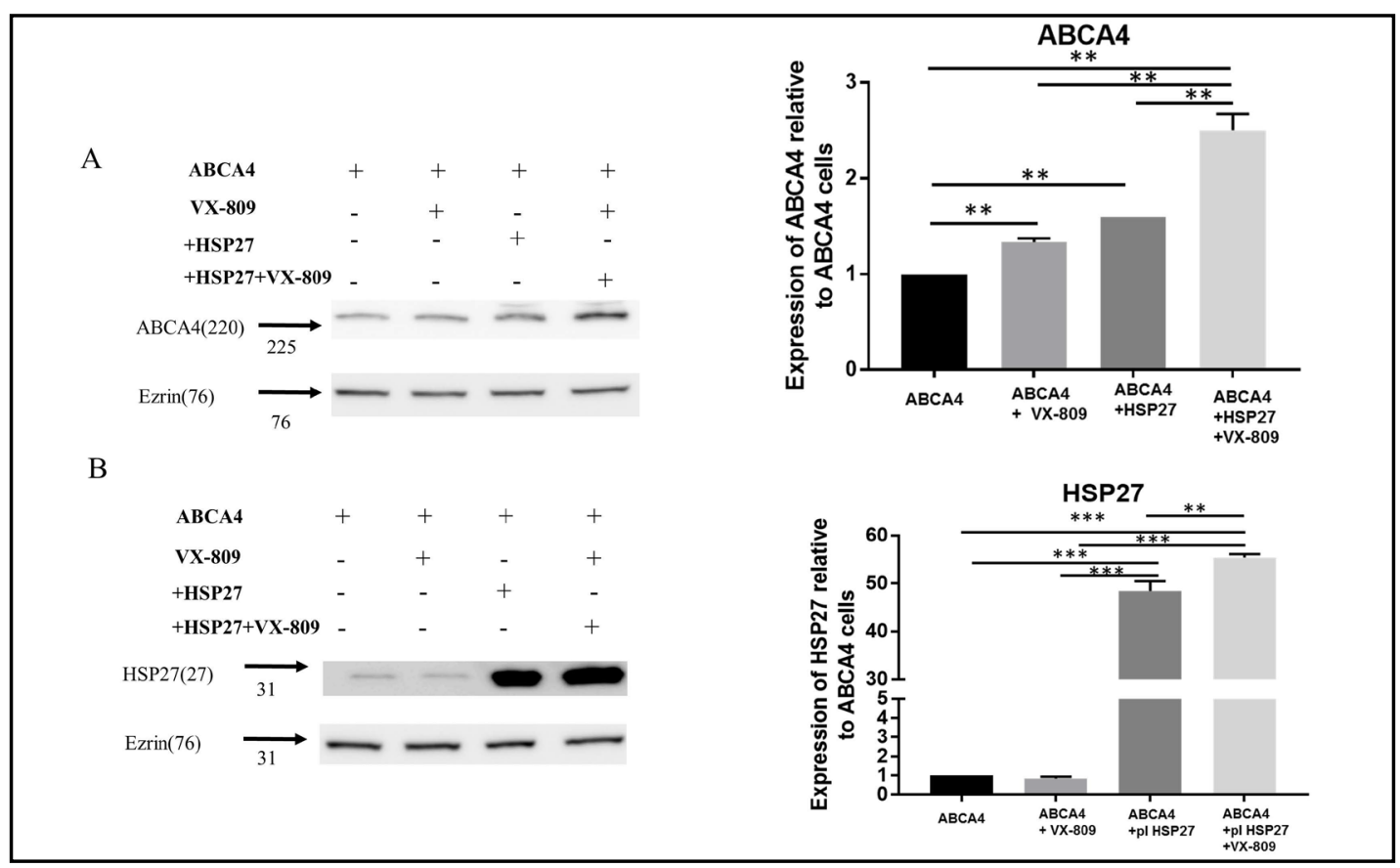

Fig. 10. Restoration of Hsp27 in cells containing wt ABCA4: (A) wt-ABCA4 containing cells were transfected with Hsp27 and treated with VX-809. (Plasmids were transfected into ABCA4 containing cell lines using Lipofectamine 2000 (Invitrogen) according to the manufacturer's instruction) The data shows that increasing Hsp27 levels causes wt-ABCA4 levels to increase. The combination of Hsp27 overexpression and addition of VX-809 are synergistic. (B) Hsp27 transfection, with or without VX-809 (20 $\mu$ M). Protein density was normalized to Ezrin. Data were represented as mean \pm SEM $(n=3)$. One way Anova was used for statistics. ${ }^{* *} \mathrm{P}<0.01$. 

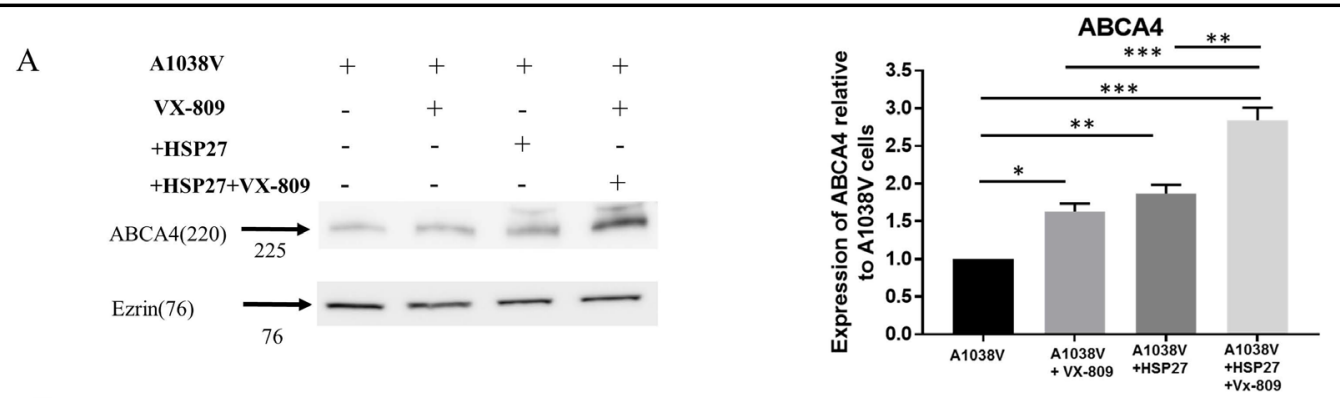

B
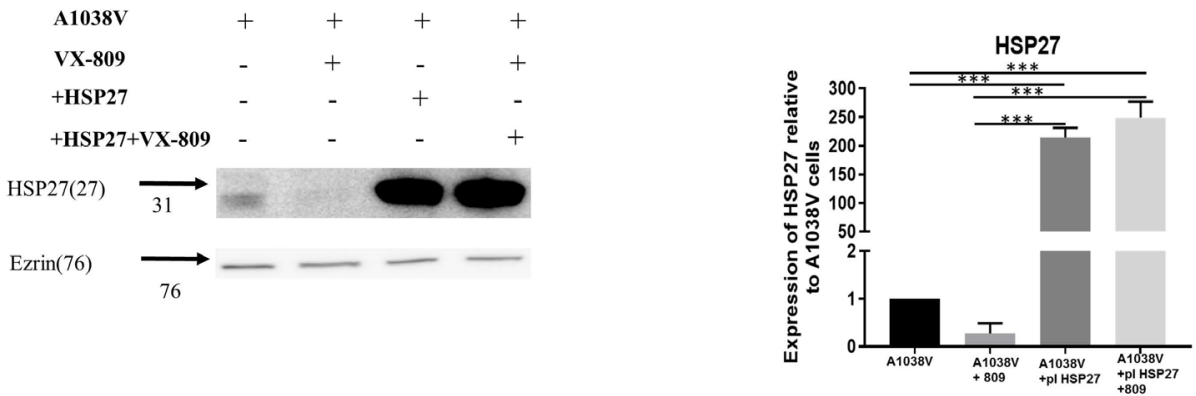

Fig. 11. Restoration of Hsp27 in cells containing A1038V ABCA4: (A) A1038V ABCA4 containing cells were transfected with Hsp27 and treated with VX-809. The data shows that increasing Hsp27 levels does not have an effect on A1038V. VX-809 does indeed cause a significant increase of approximately 2-fold in steady state levels. The combination of Hsp27 overexpression and addition of VX-809 are not synergistic. (B) Hsp27 transfection, with or without VX-809 $(20 \mu \mathrm{M})$. Protein density was normalized to Ezrin. Data were represented as mean \pm SEM $(n=3-6)$. One way Anova was used for statistics. ${ }^{* *} \mathrm{P}<0.01$.

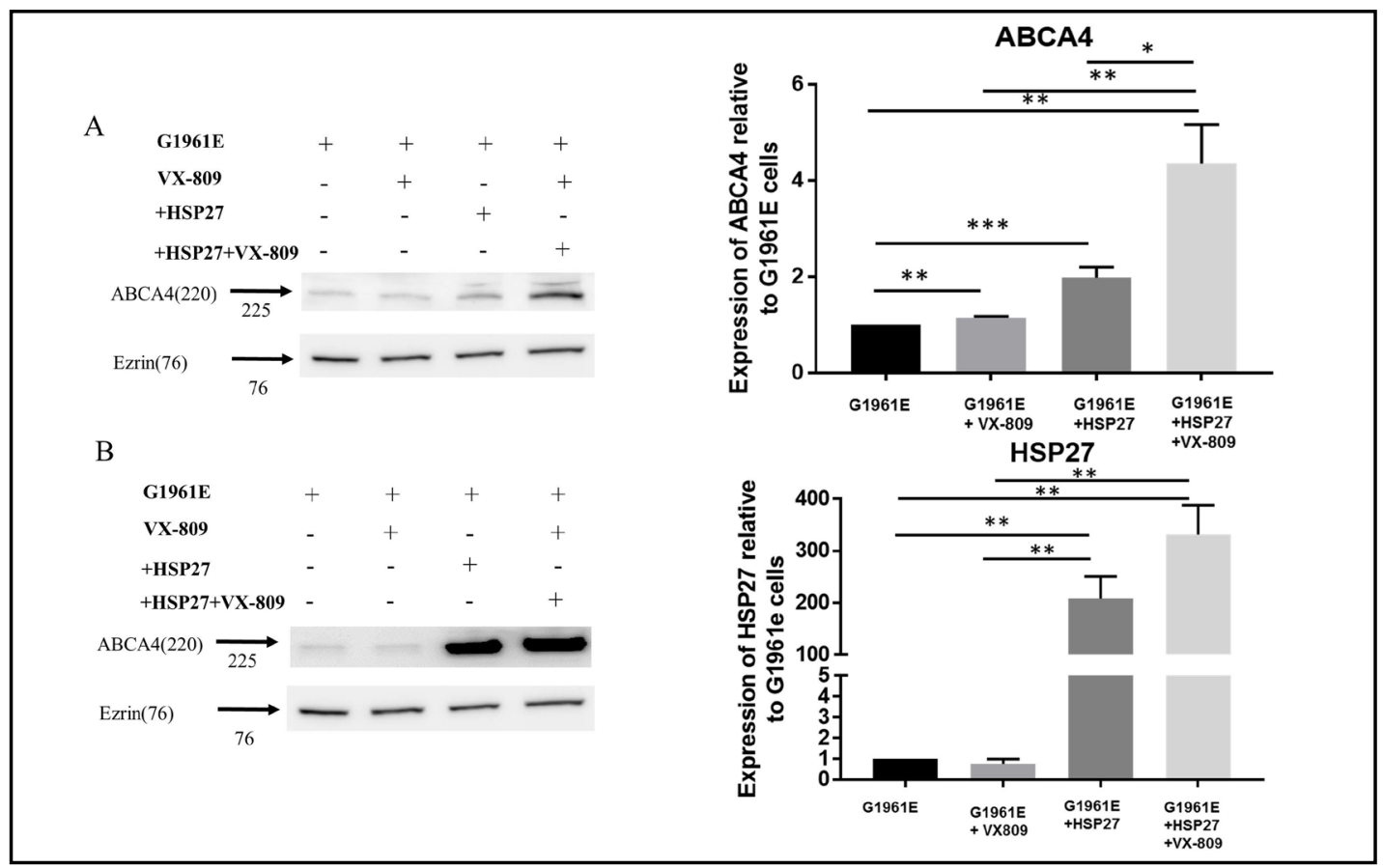

Fig. 12. Restoration of Hsp27 in cells containing G1961E ABCA4 (A) G1961E ABCA4 containing cells were transfected with Hsp27 and treated with VX-809. The data shows that increasing Hsp27 levels has no significant effect on its own. The combination of Hsp27 overexpression and addition of VX-809 are synergistic. (B) Hsp27 transfection, with or without VX-809 (20 $\mu \mathrm{M})$. Protein density was normalized to Ezrin. Data were represented as mean \pm SEM $(n=3-6)$. One way Anova was used for statistics. ${ }^{*} P<0.05$; $* * \mathrm{P}<0.01$. 


\section{Cellular Physiology Cell Physiol Biochem 2019;53:400-412 \\ \begin{tabular}{ll|l} 
and Biochemistry & $\begin{array}{l}\text { DOblished online: } 13 \text { August } 2019 \\
\text { Publions }\end{array}$ & $\begin{array}{l}\text { C } 2019 \text { The Author(s). Published by } \\
\text { Cell Physiol Biochem Press GmbH\&Co. KG }\end{array}$
\end{tabular} \\ Liu et al.: Rescuing ABCA4 Trafficking Mutants}

For G1961E, the pattern is the same but the magnitude of the results is impressive. Fig. 12A shows that increasing Hsp27, has a greater effect on increasing the steady state levels of A1038V ABCA4 than treating cells with VX-809. Increasing Hsp27 levels in the presence of VX-809 is additive causing a dramatic five- fold increase in G1961E ABCA4. These data suggest that a combined treatment which targets both ABCA4 and rescues Hsp27 levels near to those observed with wt. ABCA4 will potentially have therapeutic value. Fig. 12B shows the Hsp27 controls.

\section{Discussion}

Here we show that two Stargardt disease-causing mutations in different nucleotidebinding domains of the ABCA4 protein are degraded differently by the cell's quality control mechanism, but they can both be rescued by the small-molecule corrector VX-809, an essential component of lumacaftor, a drug that has been approved for treating CF patients [6].

\section{Parallels between ABCA4 and CFTR mutants}

The most common mutation in the NBD1 of the CFTR gene produces the $\triangle \mathrm{F} 508$ mutant protein which is a well-known trafficking mutant, recognized by endoplasmic associated degradative pathway (ERAD) and degraded. It has been suggested that A1038V in NBD1 of ABCA4 is also a processing mutant $[15,18]$. Given that ABCA4 and CFTR share $45 \%$ homology within the NBD domains [5], the question is; can we apply the information that is known for CFTR toward our understanding of ABCA4. The alanine at the site of the A1038V mutation is highly conserved [19] in the two ABC proteins. For example, in CFTR there is an identical residue at $\mathrm{A} 523$. Although there are no known $\mathrm{CF}$-causing mutations at this site, the fact that the residue is conserved makes it likely to be a critical residue for both of these $\mathrm{ABC}$ proteins.

On the other hand, G1961E is not in an area of high conservation within CFTR [19]. NBD2 of CFTR is the last domain to be translated and folded into the final structure of CFTR [20], and therefore it might be expected that ABCA4 mutations within NBD2 would behave differently from those in NBD1. For example, we previously studied two mutations in the NBD2 of CFTR, S1235R (a mild variant) and N1303K (a severe disease-causing mutation) and showed that the properties of these mutations differ from those of mutations in NBD1 [21].

It is interesting that the S1235R mutation in CFTR is part of a region similar to the one in which G1961E occurs in ABCA4. The residue in CFTR that corresponds to G1961E is S1233CFTR. Also interesting is residue R1963 in ABCA4, which corresponds to the previously studied S1235R-CFTR; note that ABCA4 already has an arginine at R1963. When we applied MG132 and tubacin to cells containing S1235R-CFTR in our previous study [21], we saw an approximately 2 - to 3 -fold increase in the $\mathrm{C}$ band and a larger, approximately 6 -fold increase in the B band of the CFTR molecule, suggesting that, like G1961E-ABCA4, S1235R-CFTR is also degraded in both proteasomes and aggresomes. S1235R-CFTR, like G1961E-ABCA4, can be rescued by small-molecule correctors. Thus, careful examination of the similarities between CFTR and ABCA4 can be exploited to provide therapeutic insights into how to treat Stargardt disease.

\section{Rescue by VX-809}

The concepts discussed above are further elucidated by the differences in the magnitude of the degree to which the two ABCA4 mutants can be rescued by VX-809. ABCA4 is a retinylidene-phosphatidylethanolamine transporter that facilitates the recycling of all-trans retinal, which originates from photoreceptors following photoexcitation [22]. Given that the presence of ABCA4 in the plasma membrane is critical for vision, we examined its surface expression by a combination of surface biotinylation and confocal microscopy. Our results show that VX-809 does indeed increase the expression of both mutant proteins at the cell 
surface, but it has a greater effect on the surface expression of G1961E than on A1038V. The observation that VX-809 increases the plasma membrane levels of both mutants suggests that VX-809 allows the mutant proteins to bypass the cells' quality control mechanism and proceed to the plasma membrane. This scenario most likely occurs as a result of increasing the stability of the mutant ABCA4 proteins by affecting ABCA4 directly. VX-809 has been classified as a Class I corrector, hypothesized to stabilize NBD1-TMD1/2 interfaces [23] of $\triangle$ F508 CFTR which is located in NBD1. Thus, the question can be asked why is VX-809 was also effective in rescuing mutations in NBD2? It is possible that mutations in NBD2 can disrupt the interactions between the transmembrane domains and NBD1 as a secondary consequence. However, it is more likely that VX-809 can influence both CFTR and ABCA4 mutations within NBD2 directly. Indeed, the greater effectiveness of VX-809 on G1961E indicates that this mutant is easier to correct and that patients with this mutation may be prime candidates for therapy involving VX-809.

The concept proposed here is that small molecules such as VX-809 can rescue the processing of trafficking mutants of ABCA4 similar to those occurring in CFTR. Even though in vitro, VX-809 rescues CFTR processing and chloride channel function (Van et al., 2006), in clinical trials with VX-809, CF patients require both a corrector and a potentiator to observe clinical benefit (Clancy et al., 2012). Thus, although we did not measure ABCA4 function directly, we would expect ABCA4 function to rescued by VX-809. The question is whether VX-809 or a similar corrector molecule will rescue enough ABCA4 activity to have a clinical impact. Since ABCA4 is a transport protein and not an ion channel [24] it is possible that a unique potentiator will have to be developed to stimulate transport following rescue of protein trafficking of the mutants studied here.

\section{Role of Hsp27}

We noted previously that two ABCA4 mutants R1108C and R1129C reduced the steady state levels of Hsp27 by approximately 75\% [5]. Here we found a similar dramatic decrease in Hsp27 by more than 50\%. Hsp27 is a low molecular weight, ATP-independent chaperone with many functions. Foremost, it is heat shock protein which binds to mutant proteins to protect them from aggregation. It also functions in the immune response and in maintaining the barrier function of epithelial cells [25]. In the $\Delta$ F508 CFTR mutation, Hsp27 is involved in targeting mutant CFTR for sumolation and ultimate degradation [26-28]. In view of this profound decrease in Hsp27 induced by the ABCA4 mutants, a similar effect of these mutants in the human eye would be expected to contribute significantly to the pathophysiology of Stargardt disease. Interestingly as we have shown previously treatment of cells with CFTR correctors causes a reduction in the steady state levels of Hsp27 [29]. Here we show that in the presence of the A1038V mutant, application of VX-809 does not restore Hsp27 to levels comparable to wt. Thus, our work supports the notion that VX-809 rescues trafficking by binding directly to the protein [30].

To test this further, we set about to increase Hsp27 levels by transfecting more into the cells. Increasing the Hsp27 levels alone increases the steady state levels of wt., A1038V and G1961E to greater extent compared to VX-809. Importantly however, is the dramatic effect noted when Hsp27 levels are increased in the presence of VX-809. In that case, the increase in steady state protein levels is higher than that observed when each is applied alone, particularly in the case of the G1961E mutant with increases approximately 5-fold. Taken together out data show that strategies to rescue ABCA4 mutants based on corrector molecule must address both the defect in ABCA4 and its interaction with the quality control network of the cell, particular with respect to Hsp27.

\section{Conclusion}

In the present study, we show that a strong understanding of the structure-function relationships of ABCA4 and CFTR can be used to study the trafficking mutants of ABCA4 and 


\section{Cellular Physiology Cell Physiol Biochem 2019;53:400-412 \\ \begin{tabular}{ll|l} 
and Biochemistry & $\begin{array}{l}\text { DOl: 10.33594/000000146 } \\
\text { Published online: } 13 \text { August } 2019\end{array}$ & $\begin{array}{l}\text { O } 2019 \text { The Author(s). Published by } \\
\text { Cell Physiol Biochem Press GmbH\&Co. KG }\end{array}$ \\
\cline { 2 - 3 }
\end{tabular} \\ Liu et al.: Rescuing ABCA4 Trafficking Mutants}

design small-molecule corrector approaches that rescue specific protein trafficking to the plasma membrane. These data provide a pathway for treatment of Stargardt disease based upon novel corrector compounds that are already being applied to the treatment of CF.

\section{Acknowledgements}

The authors acknowledge Dr. Deborah McClellan for editing the manuscript. This work was supported by NIH NHLBI grant \#1F31HL128116-01 to EASB. The research was funding by a NHLBI multi-PI grant, R01 HL122267, to LC and WBG.

Author contributions: QL, IS, EASB, MY and LC were involved in conducting the experiments. LC and WBG made substantial contributions to the conception and design of the experiments, data analysis, interpretation of data and writing the manuscript.

\section{Disclosure Statement}

The authors declare they have no conflict of interest.

\section{References}

1 Allikmets R: Simple and complex ABCR: genetic predisposition to retinal disease. Am J Hum Genet 2000;67:793-799.

2 Beharry S, Zhong M, Molday RS: N-retinylidene-phosphatidylethanolamine is the preferred retinoid substrate for the photoreceptor-specific ABC transporter ABCA4 (ABCR). J Biol Chem 2004;279:5397253979.

3 Fishman GA, Stone EM, Grover S, Derlacki DJ, Haines HL, Hockey RR: Variation of clinical expression in patients with Stargardt dystrophy and sequence variations in the ABCR gene. Arch Ophthalmol 1999;117:504-510.

4 Molday RS, Zhong M, Quazi F: The role of the photoreceptor ABC transporter ABCA4 in lipid transport and Stargardt macular degeneration. Biochim Biophys Acta 2009;1791:573-583.

5 Sabirzhanova I, Lopes Pacheco M, Rapino D, Grover R, Handa JT, Guggino WB, Cebotaru L: Rescuing Trafficking Mutants of the ATP-binding Cassette Protein, ABCA4, with Small Molecule Correctors as a Treatment for Stargardt Eye Disease. J Biol Chem 2015;290:19743-19755.

6 Mayer M: Lumacaftor-ivacaftor (Orkambi) for cystic fibrosis: behind the 'breakthrough'. Evid Based Med 2016;21:83-86.

7 Drumm ML, Wilkinson DJ, Smit LS, Worrell RT, Strong TV, Frizzell RA, Dawson DC, Collins FS: Chloride conductance expressed by delta F508 and other mutant CFTRs in Xenopus oocytes. Science 1991;254:1797-1799.

8 Genead MA, Fishman GA, Stone EM, Allikmets R: The natural history of stargardt disease with specific sequence mutation in the ABCA4 gene. Invest Ophthalmol Vis Sci 2009;50:5867-5871.

9 Riveiro-Alvarez R, Aguirre-Lamban J, Lopez-Martinez MA, Trujillo-Tiebas MJ, Cantalapiedra D, Vallespin E, Avila-Fernandez A, Ramos C, Ayuso C: Frequency of ABCA4 mutations in 278 Spanish controls: an insight into the prevalence of autosomal recessive Stargardt disease. Br J Ophthalmol 2009;93:1359-1364.

10 Fumagalli A, Ferrari M, Soriani N, Gessi A, Foglieni B, Martina E, Manitto MP, Brancato R, Dean M, Allikmets $\mathrm{R}$, Cremonesi L: Mutational scanning of the ABCR gene with double-gradient denaturing-gradient gel electrophoresis (DG-DGGE) in Italian Stargardt disease patients. Hum Genet 2001;109:326-338.

11 Cheng J, Wang H, Guggino WB: Modulation of mature cystic fibrosis transmembrane regulator protein by the PDZ domain protein CAL. J Biol Chem 2004;279:1892-1898.

12 Cheng J, Wang H, Guggino WB: Regulation of cystic fibrosis transmembrane regulator trafficking and protein expression by a Rho family small GTPase TC10. J Biol Chem 2005;280:3731-3739.

13 Cheng J, Cebotaru V, Cebotaru L, Guggino WB: Syntaxin 6 and CAL mediate the degradation of the cystic fibrosis transmembrane conductance regulator. Mol Biol Cell 2010;21:1178-1187. 
Liu et al.: Rescuing ABCA4 Trafficking Mutants

14 Amaral MD, Clarke LA, Ramalho AS, Beck S, Broackes-Carter F, Rowntree R, Mouchel N, Williams SH, Harris A, Tzetis M, Steiner B, Sanz J, Gallati S, Nissim-Rafinifa M, Kerem B, Hefferon T, Cutting GR, Goina E, Pagani F: Quantitative methods for the analysis of CFTR transcripts/splicing variants. J Cyst Fibros 2004;3:17-23.

15 Sun H, Smallwood PM, Nathans J: Biochemical defects in ABCR protein variants associated with human retinopathies. Nat Genet 2000;26:242-246.

16 Cebotaru L, Vij N, Ciobanu I, Wright J, Flotte T, Guggino WB: Cystic fibrosis transmembrane regulator missing the first four transmembrane segments increases wild type and DeltaF508 processing. J Biol Chem 2008;283:21926-21933.

17 Van GF, Straley KS, Cao D, Gonzalez J, Hadida S, Hazlewood A, Joubran J, Knapp T, Makings LR, Miller M, Neuberger T, Olson E, Panchenko V, Rader J, Singh A, Stack JH, Tung R, Grootenhuis PD, Negulescu P: Rescue of DeltaF508-CFTR trafficking and gating in human cystic fibrosis airway primary cultures by small molecules. Am J Physiol Lung Cell Mol Physiol 2006;290:L1117-L1130.

18 Brodsky JL: Chaperoning the maturation of the cystic fibrosis transmembrane conductance regulator. Am J Physiol Lung Cell Mol Physiol 2001;281:L39-42.

19 Lewis HA, Buchanan SG, Burley SK, Conners K, Dickey M, Dorwart M, Fowler R, Gao X, Guggino WB, Hendrickson WA, Hunt JF, Kearins MC, Lorimer D, Maloney PC, Post KW, Rajashankar KR, Rutter ME, Sauder JM, Shriver S, Thibodeau PH, et al.: Structure of nucleotide-binding domain 1 of the cystic fibrosis transmembrane conductance regulator. EMBO J 2004;23:282-293.

20 Rosser MFN, Grove DE, Chen LL, Cyr DM: Assembly and Misassembly of Cystic Fibrosis Transmembrane Conductance Regulator: Folding Defects Caused by Deletion of F508 Occur Before and After the Calnexindependent Association of Membrane Spanning Domain (MSD) 1 and MSD2. Mol Biol Cell 2008;19:45704579.

21 Rapino D, Sabirzhanova I, Lopes-Pacheco M, Grover R, Guggino WB, Cebotaru L: Rescue of NBD2 mutants N1303K and S1235R of CFTR by small-molecule correctors and transcomplementation. PLoS One 2015;10:e0119796.

22 Quazi F, Lenevich S, Molday RS: ABCA4 is an N-retinylidene-phosphatidylethanolamine and phosphatidylethanolamine importer. Nat Commun 2012;3:925.

23 Okiyoneda T, Barriere H, Bagdany M, Rabeh WM, Du K, Hohfeld J, Young JC, Lukacs GL: Peripheral protein quality control removes unfolded CFTR from the plasma membrane. Science 2010;329:805-810.

24 Molday RS, Zhang K: Defective lipid transport and biosynthesis in recessive and dominant Stargardt macular degeneration. Prog Lipid Res 2010;49:476-492.

25 Seit-Nebi AS, Gusev NB: Versatility of the small heat shock protein HSPB6 (Hsp20). Cell Stress Chaperones 2010;15:233-236.

26 Ahner A, Gong X, Schmidt BZ, Peters KW, Rabeh WM, Thibodeau PH, Lukacs GL, Frizzell RA: Small heat shock proteins target mutant cystic fibrosis transmembrane conductance regulator for degradation via a small ubiquitin-like modifier-dependent pathway. Mol Biol Cell 2013;24:74-84.

27 Ahner A, Gong X, Frizzell RA: Cystic fibrosis transmembrane conductance regulator degradation: cross-talk between the ubiquitylation and SUMOylation pathways. FEBS J 2013;280:4430-4438.

28 Zhang H, Peters KW, Sun F, Marino CR, Lang J, Burgoyne RD, Frizzell RA: Cysteine string protein interacts with and modulates the maturation of the cystic fibrosis transmembrane conductance regulator. J Biol Chem 2002;277:28948-28958.

29 Lopes-Pacheco M, Boinot C, Sabirzhanova I, Morales MM, Guggino WB, Cebotaru L: Combination of Correctors Rescue DeltaF508-CFTR by Reducing Its Association with Hsp40 and Hsp27. J Biol Chem 2015;290:25636-25645.

30 Wang Y, Loo TW, Bartlett MC, Clarke DM: Correctors promote maturation of cystic fibrosis transmembrane conductance regulator (CFTR)-processing mutants by binding to the protein. J Biol Chem 2007;282:3324733251. 